Rev. Elev. Méd. vét. Pays trop., 1978, 31 (1) : 11-19.

\title{
Culture du virus claveleux sur cellules : Application à la vaccination et au contrôle de l'immunité
}

\author{
par J. RAMISSE $\left({ }^{*}\right)$, J. ASSO $(* *)$, A. HASSANI $(* * *)$ \\ O. ANANE $(* * *)$ et J. JEMLI $(* * *)$
}

\begin{abstract}
RESUMẼ
Dans cette publication sont étudiées quelques propriétés du virus claveleux cultivé sur cellules in vitro: E. C. P., titre, innocuité, pouvoir immunisant, induction d'anticorps sériques spécifiques.
\end{abstract}

L'étude de la multiplication du virus claveleux en cultures cellulaires remonte à une vingtaine d'années $(2,5)$. Le but de cette technique était de mettre au point un vaccin vivant, atténué, inoffensif et suffisamment immunogène. Différents essais se sont déroulés pendant ces 20 dernières années. Les résultats en sont rapportés dans les publications ci-après référencées $(2,1,3$, $4,5,6,7)$. Ces résultats sont encourageants et il semble que, désormais, on puisse disposer d'un vaccin vivant valable contre la clavelée.

En Tunisie, à ce jour, la clavelée existe ; mais elle n'y est pas économiquement très grave. Car du fait de la prophylaxie généralisée (vaccination annuelle à l'aide de vaccin inactivé), l'immunité collective obtenue empêche l'éclosion de foyers nombreux ou très meurtriers.

En vue de faciliter la diffusion, la conservation et l'emploi d'un vaccin vivant anti-claveleux, l'Institut de Recherches Vétérinaires de Tunisie a

(*) I. E. M. V. T. 10, rue Pierre-Curie, 94700 MaisonsAlfort, France, détaché à l'I. R. V. T. (Tunis).

(**) Laboratoire de Virologie, I. N. R. A., 78850 Thiverval-Grignon.

(***) Directeur de l'I. R. V. T., La Rabta, Tunis, République de Tunisie.

$(* * * *)$ I. R. V. T., Tunis. décidé d'étudier la mise au point, sur place, d'une expérimentation portant sur l'atténuation de souches locales, ou l'utilisation de souches déjà atténuées. A la suite, devait être abordée l'étude des caractères de l'immunité induite (nature, durée).

Les méthodes employées pour réaliser ce travail, ainsi que les résultats obtenus sont exposés dans la présente publication.

\section{LE MATÉRIEL}

\section{Les souches de virus}

Une souche virulente locale isolée en 1974 (souche Zaghouan) et deux souches atténuées $\left(^{*}\right.$ ) (A1 et $\mathrm{A} 2$ ) ont été utilisées à cet effet. La souche virulente est conservée au froid $\left(-20^{\circ}\right)$ sous forme de lymphe claveleuse. Les souches atténuées ont été lyophilisées avant leur passage sur cellules et leur titre infectieux en culture cellulaire est de l'ordre de $10^{4} \mathrm{DICT}_{50} / \mathrm{ml}$.

(*) Nous remercions nos confrères ASSO et PERREAU d'avoir bien voulu nous procurer ces souches. 


\section{Les cultures cellulaires}

Parmi tous les systèmes cellulaires essayés, les 2 qui nous ont donné les meilleurs résultats sont : les cellules testiculaires de jeune agneau et les cellules rénales de fœtus ovin. Dans tous les cas, il s'agissait de cultures de première explantation. Nous n'utilisions toutefois que les subcultures des premiers ou deuxièmes passages.

Pour la multiplication des cellules, nous avons travaillé avec les milieux classiques (HANKS à l'hydrolysat de lactalbumine, 199, M. E. M.). Pour des raisons de commodité et d'économie, le sérum de veau importé a été remplacé par du sérum de bœuf préparé à partir de sang récolté à l'abattoir de Tunis.

\section{Les moutons}

Pour les diverses expérimentations mises en place, des agneaux de 5 à 6 mois ont été utilisés. Ils étaient de race locale (barbarine), ou importés (races européennes). Dans chaque expérience la réceptivité des agneaux à la clavelée a été démontrée, avant toute inoculation, par l'absence d'anticorps neutralisants sériques.

\section{ÉTUDE DE LA SOUCHE DE Clavelée SAUVAGE (SOUCHE ZAGHOUAN)}

Cette souche demeure virulente pour le mouton, et elle sert à la préparation du vaccin inactivé. Elle provoque chez le mouton réceptif de fortes lésions locales (avec escarrification et gangrène), accompagnées de généralisation (virémie, fièvre élevée, anorexie, prostration, éruptions secondaires), avec le plus souvent une issue fatale.

Elle a été passée en cultures cellulaires dans l'espoir d'obtenir une atténuation rapide et stable.

La technique de passage est classique : les cultures de cellules rénales ou testiculaires ovines sont inoculées avec des suspensions virulentes provenant de lymphe claveleuse ou de broyats tissulaires (dilués au $1 / 10$ ou au 1/100). Après une adsorption suffisante ( 1 à $2 \mathrm{~h}$ à $37^{\circ}$ ), l'inoculum est rejeté, la nappe cellulaire lavée et, après addition du milieu d'entretien, les cellules ino- culées sont incubées à $37^{\circ}$ pendant 8 à 10 jours (ou davantage si nécessaire), jusqu'à ce qu'apparaisse l'effet cytopathogène viral. Lorsque le virus se multiplie convenablement sur cellules, il est titré par la méthode des dilutions terminales, soit en cultures cellulaires, soit sur moutons.

La première série de passages a abouti à isoler en culture cellulaire, à partir du virus sauvage, une souche virale cytopathogène, de titre élevé (de l'ordre de $10^{7} \mathrm{DICT}_{50} / \mathrm{ml}$ ), neutralisée par l'antisérum claveleux. Mais l'inoculation au mouton des $10^{\circ}$ et $14^{\mathrm{e}}$ passages nous a montré qu'il ne s'agissait plus de virus claveleux. La souche inoculée ne provoquait pas de lésion cutanée et ne protégeait plus contre l'infection expérimentale virulente.

La deuxième série de passages de la souche sauvage, contrôlée parallèlement sur mouton, a donné des résultats positifs quant à la multiplication du virus en culture cellulaire : effet cytopathogène régulier croissant, accéléré au fur et à mesure des passages, Cet E. C. P. est plus régulier et plus lisible sur cellules testiculaires que sur cellules rénales. Les titres sont peu élevés: $10^{4}-10^{4,5}$ DICT $_{50} / \mathrm{ml}$, selon l'état des cellules. Le virus conserve un pouvoir pathogène important pour le mouton. Au $10^{\mathrm{e}}$ passage en culture cellulaire, il provoque chez le mouton inoculé par voie intra-dermique la formation de nodules et même de tumeurs inflammatoires locales qui s'escarrifient, se nécrosent et se gangrènent. Dans la plupart des cas, il y a généralisation et mort des animaux. Ces réactions s'observent quelles que soient les doses de virus inoculées (1/10-1/1 000 de $\mathrm{ml})$.

Si les sujets survivent, ils sont résistants à une épreuve virulente expérimentale.

Enfin, ce virus de culture cellulaire est également neutralisé par les anti-sérums claveleux de diverses origines $(*)$, ainsi que par des sérums de moutons guéris de clavelée.

Constatant que les passages en cultures cellulaires de la souche tunisienne sauvage de clavelée n'étaient pas rapidement suivis d'atténuation, nous avons préféré, pour gagner du temps, abandonner cette option et travailler avec des souches déjà atténuées.

(*) Nous remercions les Directeurs de l'Institut Razi (Téhéran), et de l'Institut Pasteur d'Alger, pour avoir bien voulu nous fournir ces sérums. 


\section{COMPORTEMENT \\ DES SOUCHES ATTÉNUÉES \\ EN CULTURES CELLULAIRES}

1. Effet cytopathogène et titres en cultures cellulaires

Les deux souches atténuées (A1 et A2) se sont comportées d'une manière identique sur cellules. Les passages ont été faits, en règle générale, sur cellules testiculaires d'agneau.

Le virus inoculé au 1/10 (ou au 1/100) produit un effet cytopathogène au bout de 4 à 6 jours, qui s'accentue jusqu'aux $12^{\mathrm{e}}-15^{\mathrm{e}}$ jours. L'aspect morphologique de cet E. C. P. est comparable à celui de la souche sauvage. On peut l'observer sur les clichés $n^{\text {os }} 3$ et 4 . A titre de comparaison, les photos 1 et 2 représentent des cultures normales de cellules rénales et testiculaires ovines.

On notera un E. C. P. plus avancé sur les cellules rénales, avec la présence d'inclusions cytoplasmiques entourées d'une auréole. L'E. C. P. est cependant plus lisible à la longue, et plus régulier, avec les cellules testiculaires qui, à notre avis, permettent un meilleur titrage. Les
Photo $\mathrm{n}^{\circ}$ 1. - Culture de celfules rénales de foetus ovin (H. E.).

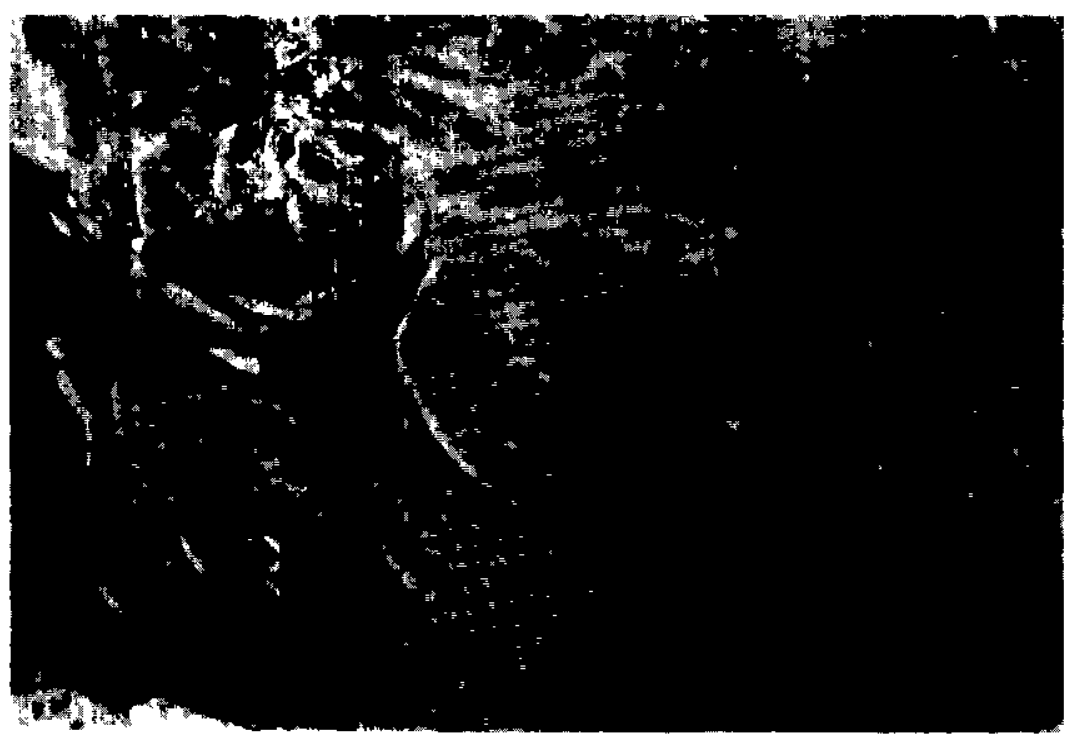

Photo $\mathrm{n}^{\circ} 2$. - Culture de cellules testiculaires d'agneau (H. E.). 


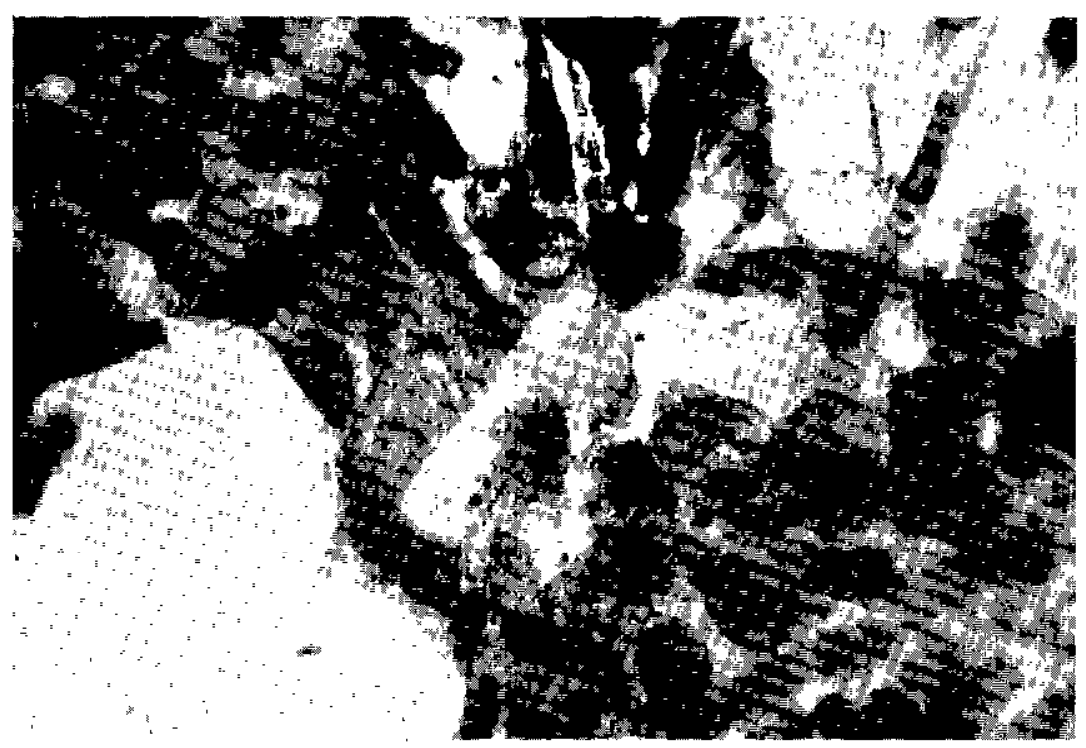

Photo $\mathrm{n}^{\circ}$ 3. - Culture de cellules rénales de foetus ovin infectée avec le virus de la clavelée. Aspect de l'E. C. P. (Inclusions cytoplasmiques (H. E.).

Photo $\mathrm{n}^{0} 4$, - Culture de cellules testiculaires de mouton infectée avec le virus de la clavelée. Début de l'E. C. P. (H. E.).

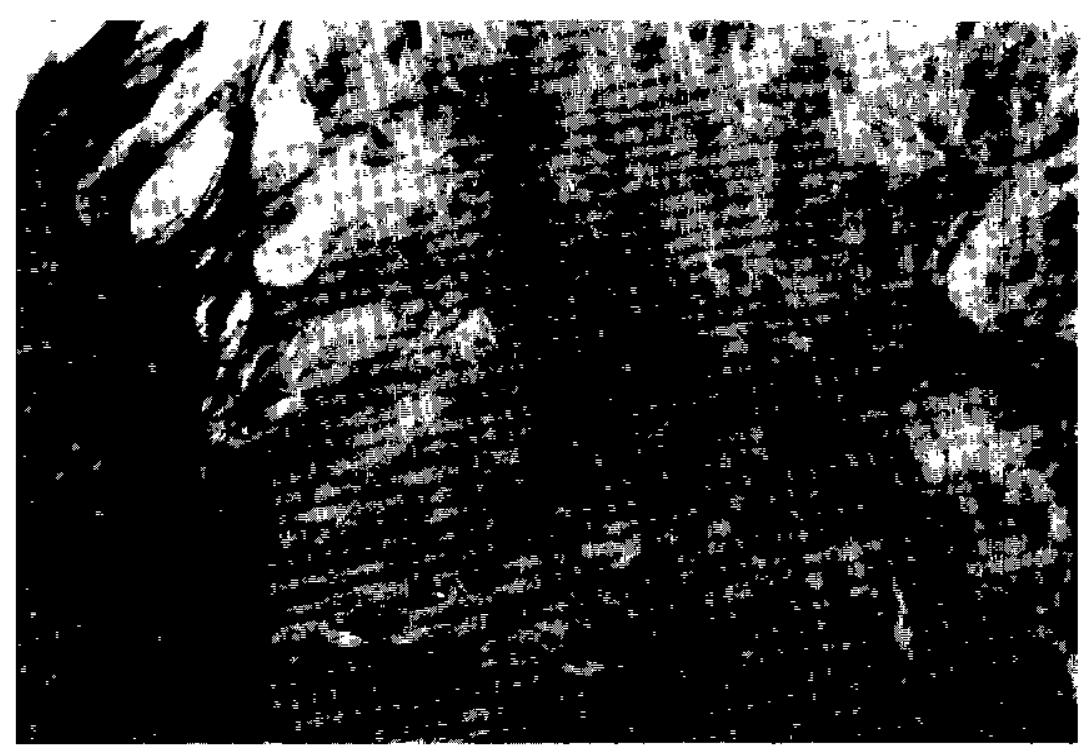

cellules testiculaires se maintenant mieux que les cellules rénales, il est également préférable de les utiliser pour les séro-neutralisations.

Les titres obtenus sur les deux systèmes sont pratiquement identiques (de l'ordre de $4 \times 10^{4,5}$ à $10^{5,5} \mathrm{DICT}_{50} / \mathrm{ml}$ ). Les cultures de mauvaise qualité donnent un titre moindre, pouvant s'abaisser jusqu'à $10^{2,5} \mathrm{DICT}_{50} / \mathrm{ml}$. Les passages en série n'augmentent pas le titre.

Les résultats des séro-neutralisations confirment l'identité des deux souches atténuées. Celles-ci sont neutralisées totalement par les antisérums claveleux d'Iran, d'Alger, de Tunis, et par les sérums de moutons guéris de clavelée. Elles ne sont évidemment pas neutralisées par les sérums normaux.

\section{Effet des conditions de culture sur le titre du virus}

Influence de la nature des cellules support : la meilleure replication du virus claveleux s'obtient sur cellules rénales ou testiculaires ovines. Le titre est à peu près identique sur les deux systèmes. 
Mais la lecture des titrages et séro-neutralisation est, nous l'avons déjà dit, plus facile avec les cellules testiculaires.

- Influence du milieu d'entretien : l'addition de sérum de veau au milieu d'entretien, à faible concentration ( 1 à 2 p. 100) ne modifie pas le titre final.

- Influence de la durée d'incubation des cultures : il faut attendre pour avoir un bon titre que l'E. C. P. soit suffisamment avancé. Le titre infectieux augmente avec la manifestation de $1^{\prime} E$. C. P. jusqu'aux $6^{e}$ et $8^{e}$ jours après l'inoculation des cultures. La congélation des cultures lysées au sortir de l'étuve, et avant la récolte du virus, n'interviendra que lorsque l'E. C. P. aura détruit au moins les $3 / 4$ de la nappe cellulaire. Le virus produit en culture cellulaire résiste assez bien à la température de l'étuve et il n'est pas rapidement inactivé après sa libération hors des cellules.

- Influence du pH des cultures : Il n'y a pas de variation de titre dans les limites supportables par les cellules (pH entre 6,8-7,8).

En somme, ce virus apparaît assez résistant, avec une réplication lente, un titre peu élevé qui dépend essentiellement de l'état des cellules support.

\section{OBSERVATIONS SUR LA CONSERVATION DU VIRUS CLAVELEUX}

La répétition des congélations et décongélations a un effet néfaste sur le virus claveleux, encore plus marqué d'ailleurs sur le virus de culture cellulaire que sur celuı des tissus ou de la lymphe. Titré sur mouton, après 3 ou 4 congélations et décongélations, le virus perd 4 logs d'infectiosité. De même pour la souche atténuée cultivée sur cellules. Son titre à la récolte étant, par exemple, de $4 \times 10^{5} \mathrm{DICT}_{50} / \mathrm{ml}$, il n'est plus que de $4 \times 10^{3,5}$ après un mois de conservation à $-20^{\circ}$ et une deuxième décongélation. Un mois plus tard, après une troisième décongélation, le titre a baissé à $2 \times 10^{3} .15$ jours plus tard, après une quatrième décongélation, le titre descend à $4 \times 10^{2}$.

Par contre, conservé à $-80^{\circ}$ le virus se maintient intact dans la lymphe ou les tissus, au moins pendant deux ans.

La lyophilisation conserve bien le virus. Les essais réalisés à Tunis nous ont fait choisir comme excipient le plus favorable le lait écrémé, à volume égal avec la suspension virale. Pendant l'opération de lyophilisation, il y a une baisse de titre, mais qui est inférieure à $0,5 \log 10 \ldots$ le procédé paraît donc convenir pour la préparation de stocks de vaccin devant être conservés et transportés dans les diverses régions du pays.

\section{INOCULATION AU MOUTON : INNOCUITÉ ET TITRAGE DU VIRUS}

Les animaux de laboratoire ne sont pas réceptifs au virus claveleux. L'étude expérimentale du virus ne peut donc être conduite qu'avec l'animal naturellement sensible, le mouton, ou avec des systèmes tels que les cultures cellulaires.

L'effet sur le mouton dépend de plusieurs facteurs parmi lesquels la virulence de la souche, et la race du sujet sont les plus importants.

L'innocuité d'une souche est basée sur l'absence de symptômes généraux et locaux chez l'animal inoculé. Les titrages in vivo consistent à inoculer au mouton par voie intra-dermique sur le flanc préalablement rasé et nettoyé, des dilutions croissantes de virus sous le volume de $0,5 \mathrm{ml}$ (ou $1 \mathrm{ml}$ ). Les nodules locaux apparaissant 4 à 8 jours plus tard indiquent la présence du virus dans les dilutions correspondantes. Les titrages, in vitro, ainsi qu'il a déjà été indiqué se font par la méthode des dilutions terminales avec observation de l'E. C. P. produit sur cellules testiculaires d'agneau. Les résultats obtenus varient selon les souches, les échantillons et l'état des cellules.

\section{Innocuité}

- Variable selon les souches:

- La souche tunisienne sauvage a un pouvoir pathogène élevé, et cette virulence n'est pas atténuée après une dizaine de passages sur cellules. Cela n'est pas pour surprendre, puisqu'il a été déjà établi (6) qu'il fallait attendre le $50^{\mathrm{e}}$ voire le $100^{\mathrm{e}}$ passage sur cellules pour obtenir une atténuation stable et suffisante du virus claveleux.

- La souche atténuée (Al) ne provoque que des lésions locales plus ou moins discrètes, sans généralisation. Les nodules inflammatoires locaux ont un diamètre de 1 à $2 \mathrm{~cm}$, sans tendance à l'escarrification et à la nécrose. On note une légère hyperthermie peu durable. 
- Variable selon la race de mouton :

- Les moutons tunisiens (race barbarine) sont naturellement moins sensibles au virus claveleux que les moutons européens. Chez eux l'inoculation de la souche atténuée Al ne provoque pratiquement pas de fièvre, et les nodules réactionnels sont très petits.

- Chez les agneaux européens, la souche A1 produit des réactions locales plus fortes (nodules de $2 \mathrm{~cm}$, durs, inflammatoires avec lente résorption). L'inoculation est suivie d'une hyperthermie modérée, sans trouble de l'état général.

- La voie d'inoculation ne modifie pas la virulence ni le titre. Le virus étant dermotrope, il sera inoculé habituellement par la voie transcutanée. Les réactions sont identiques, que l'inoculation soit sous-cutanée ou intra-dermique. Peut-être les titrages sont-ils plus lisibles après inoculation intra-dermique.

La réaction locale diminue avec la dose. Aux concentrations les plus fortes $\left(10^{-1}-10^{-4}\right)$, les nodules sont plus précoces et plus gros. A la limite du pouvoir infectieux $\left(10^{-6}-10^{-7}\right)$, les nodules sont plus discrets et ne se nécrosent pas.

\section{Titrage in vivo. Comparaison avec Ies titres in vitro}

- Affaiblissement du titre infectieux lors des premiers passages en culture cellulaire. La réduction du titre infectieux lors des premiers passages est importante. Nous l'avons vérifié pour la souche sauvage tunisienne. Alors que le titre initial de la lymphe est de $10^{7} \mathrm{DMI} / \mathrm{ml}$ (pour le mouton), la suspension virale de culture cellulaire ne contient que $10^{4,5} \mathrm{DMI} / \mathrm{ml}$.

- Relation entre les titres in vivo et in vitro. Pour une même suspension virale de culture cellulaire, les titres obtenus in vivo, sur mouton, sont toujours supérieurs à ceux déterminés en culture cellulaire. Ainsi, la souche atténuée A1 titrée sur mouton, après la récolte, atteint régulièrement $10^{6}$ à $10^{7} \mathrm{DMI} / \mathrm{ml}$ de suspension virale, alors que son titre en culture cellulaire n'est que de $4 \times 10^{4,5}, 4 \times 10^{5,5}$ DICT $_{50} / \mathrm{ml}$. Il y a un rapport assez constant entre les titres in vivo et in vitro. Ce qui permet d'évaluer approximativement combien de DMI représente 1 DICT $_{50}$.

\section{POUVOIR IMMUNISANT DE LA SOUCHE ATTÉNUÉE A1}

Cette propriété a été étudiée en vaccinant des moutons réceptifs, et en les infectant expérimentalement ensuite après des délais variables.
Avant l'inoculation des animaux, l'absence d'anticorps sériques a été vérifiée (prises de sang et séro-neutralisation en cultures cellulaires).

Trois points ont été abordés :

- détermination de la dose minimale vaccinale ;

- les modalités de l'épreuve pour apprécier le degré d'immunité ;

- la durée de l'immunité.

\section{Dose minimale vaccinale}

Le virus de culture cellulaire, préalablement titré sur cellules, est inoculé sous le volume de $1 \mathrm{ml}$, par voie sous-cutanée, et à dilutions croissantes $\left(10^{-1}\right.$ à $\left.10^{-7}\right)$ à des moutons sensibles. Chaque groupe d'agneau reçoit une dilution du virus-vaccin. Les réactions locales et éventuellement générales, sont notées. Après 1 mois d'observation, les animaux sont éprouvés, en même temps que des témoins, avec une dose uniforme de virus virulent, par la voie souscutanée.

Un premier essai, fait avec des agneaux tunisiens, a montré que le virus de culture ( $\mathrm{A} 1$, $4^{\circ}$ passage), protège au moins, jusqu'à la dilution $10^{-5}$, contre l'infection expérimentale.

Un deuxième essai, comportant des agneaux importés, a été réalisé avec le $7^{\mathrm{e}}$ passage en culture de la souche A1. Le titre infectieux était de $4 \times 10^{4,5} \mathrm{DICT}_{50} / \mathrm{ml}$. Les dilutions de virusvaccin allaient de $10^{-1}$ à $10^{-7}$. Des prises de sang ont été faites pendant la période d'observation pour vérifier le niveau des anticorps neutralisants sériques.

Le deuxième essai a démontré l'efficacité protectrice du virus de culture jusqu'à la dilution $10^{-7}$. Cela correspond à la limite du pouvoir infectieux du virus pour le mouton. Autrement dit à $1 \mathrm{DMr}$, et à environ $1 / 100 \mathrm{de} \mathrm{DICT}_{50}$.

Il faut noter que les agneaux immunisés avaient tous présenté une réaction locale au point d'inoculation du virus-vaccin.

D'autre part, les sérums des agneaux prélevés après vaccination étaient neutralisants jusqu'au $1 / 8^{\mathrm{e}}$ ou $1 / 16^{\mathrm{e}}$ vis-à-vis de $100 \mathrm{DICT}_{50}$ de virus.

\section{Modalités de l'épreuve pour apprécier le degré d'immunité}

D'après les données de la littérature, la technique d'épreuve expérimentale dans la clavelée ne paraît pas bien codifiée. Elle varie selon les 
auteurs. Le délai entre la vaccination et l'épreuve va de 12 jours à 3 semaines. La quantité de virus d'épreuve n'est pas indiquée avec précision ( $1 \mathrm{ml}$ de lymphe, $1 / 10 \mathrm{ml}$ de broyat, etc.). Il est parfois mentionné cependant, une dose de 50 , 1000 ou 10000 DMI. La voie d'inoculation est aussi très variable : sous-cutanée, intra-veineuse, intra-dermique, respiratoire.

Il nous a paru utile de préciser les conditions de l'épreuve et, pour cela, nous avons adopté une technique inspirée du test de Habel (dans la rage), et du travail de Sureau (Alger, 1967). Pratiquement, cela consiste à faire un titrage comparatif de la souche virulente d'épreuve, simultanément sur les sujets vaccinés et sur les témoins. Le titrage est réalisé sur les moutons, par injections intra-dermiques (flanc) sous le volume de $0,5 \mathrm{ml}$, de dilutions croissantes du virus d'épreuve, $\left(10^{-1}-10^{-7}\right)$. L'apparition de nodules inflammatoires aux points d'injections confirme la présence de virus infectieux dans l'inoculum correspondant - on apprécie ainsi la limite du pouvoir infectieux du même virus virulent sur les vaccinés et sur les témoins. La différence en logs de 10 entre le titre sur témoins et le titre sur vaccinés exprime le degré d'immunité. S'il n'y a pas de différence, il n'y a pas d'immunité.

Les résultats sont les suivants :

- Titre infectieux de la souche d'épreuve sur agneaux témoins : variable selon l'échantillon de virus.

Il va de $10^{4}$ à $10^{7} \mathrm{DMI} / \mathrm{ml}$.

- Titre infectieux de la souche d'épreuve sur agneaux vaccinés : variable également selon l'échantillon et aussi selon le moment de l'épreuve.

Il va de 0 à $10^{3} \mathrm{DMI} / \mathrm{ml}$.

- La différence entre les titres obtenus simultanément sur témoins et vaccinés indique le degré de protection (indice d'immunité) : elle varie de 3 à $7 \log 10$. Or selon Sureau (6) une différence de 3 logs 10 suffit pour affirmer l'efficacité d'un vaccin anti-claveleux.

\section{Contrôle de la durée de l'immunité}

Un lot d'agneaux importés a été vacciné avec une dose déterminée de virus de culture : souche $\mathrm{A} 1,8^{\mathrm{e}}$ passage sur cellules testiculaires, $1 \mathrm{ml}$ de la suspension à $10^{-4}$, ce qui correspond approximativement à $10 \mathrm{DICT}_{50}$ et $1000 \mathrm{DMI}$. La vaccination a été faite en sous-cutanée, en arrière de l'épaule. Avant et après vaccination, des prélèvements de sang ont été exécutés afin de contrôler le pouvoir neutralisant des sérums. Chaque mois après la vaccination une partie du lot vacciné était éprouvée, en même temps que des témoins, avec du virus virulent. Avant chaque épreuve, le niveau des anticorps sériques était contrôlé par séro-neutralisation. Les épreuves ont été réalisées selon les modalités précédemment exposées.

Pour diverses raisons, l'expérimentation n'a pu malheureusement se poursuivre au-delà de 6 mois. Les résultats en sont relatés ci-après:

- Réactions post-vaccinales :

générales : nulles,

locales : nodules inflammatoires locaux gros comme une noix, guérison spontanée.

- Présence d'anticorps neutralisants dans les sérums des agneaux :

avant vaccination : tous négatifs, après vaccination: présence d'anticorps neutralisants, les titres en sont donnés plus loin.

- Evolution de l'immunité en fonction du temps :

TABLEAL $N^{\circ} I$ - Durée de 1 'immuni té.

\begin{tabular}{|c|c|c|c|}
\hline $\begin{array}{c}\text { Délai entre vaccination } \\
\text { et épreuve (mois) }\end{array}$ & $\begin{array}{c}\text { Ti tre du virus d'épreuve } \\
\text { sur les vaccinés }\end{array}$ & $\begin{array}{c}\text { Titre du virus d'épreuve } \\
\text { sur les témoins }\end{array}$ & $\begin{array}{c}\text { Degré de protection } \\
\text { Différence en logs } \\
\text { entre les titres }\end{array}$ \\
\hline 1 & 0 & $10^{7}$ & 7 \\
\hline 2 & 0 & $10^{6}$ & 6 \\
\hline 3 & 0 & $10^{6}$ & 6 \\
\hline 4 & $10^{3}$ & $10^{6}$ & 3 \\
\hline 5 & $10^{2}$ & $10^{5}$ & 3 \\
\hline 6 & 0 & $10^{4}$ & 4 \\
\hline
\end{tabular}


A la lecture de ce tableau, on remarquera :

- que les titres du virus d'épreuve vont en diminuant (effet de la conservation et surtout des congélations et décongélations répétées) ;

- que le degré de protection (indice d'immunité) demeure suffisant au moins pendant 6 mois (durée de notre expérimentation);

- que l'indice d'immunité, très élevé au début, s'affaiblit à partir du $4^{\mathrm{e}}$ mois.

\section{RELATION ENTRE L'IMMUNITÉ ET LE NIVEAU D'ANTICORPS SÉRIQUES}

Parallèlement à l'étude de la durée d'immunité, l'évolution du titre des anticorps sériques a été suivie par des séro-neutralisations en cultures cellulaires. La technique peut être ainsi résumée : virus constant $\left(100 \mathrm{DICT}_{50}\right)$, sérum variable (dilution $\mathrm{du} 1 / 5^{\mathrm{e}}$ à $1 / 20^{\mathrm{e}}$ et plus), neutralisation $2 \mathrm{~h}$ à $37^{\circ}$, système révélateur: cellules testiculaires d'agneau, observation pendant 15 jours. Le titre d'un sérum, dans les conditions de notre expérience, est la dilution limite qui neutralise totalement $100 \mathrm{DICT}_{50} \mathrm{de}$ virus.

1. Résultats des séro-neutralisations en cultures cellulaires

- Les sérums des agneaux neufs, non inoculés, ne contiennent pas d'anticorps neutralisants anti-claveleux. Ceci quelle que soit la race.

- Les sérums des agneaux vaccinés avec le virus vaccin $\mathrm{A} 1$ contiennent des anticorps neutralisants. Les titres de ces sérums varient peu pendant les 6 mois suivant la vaccination. Au premier titrage, 15 jours après vaccination, le titre moyen est compris entre $1 / 5^{\circ}$ et $1 / 10^{\circ}$. A partir de 1 mois, et jusqu'au $4^{\mathrm{e}}$ mois après vaccination, les titres moyens contrôlés tous les mois, avant épreuve, sont compris entre $1 / 10^{\mathrm{e}}$ et $1 / 20^{\circ}$. Après le $4^{\mathrm{e}}$ mois, les titres s'abaissent et se situent à nouveau entre $1 / 5^{\mathrm{e}}$ et $1 / 10^{\mathrm{e}}$.

- Les sérums prélevés après épreuve donnent des résultats comparables à ceux qui précèdent. Peut-être les conditions de notre expérience ne sont-elles pas assez fines pour distinguer des différences de titre entre les sérums des vaccinés et ceux des éprouvés. En tous cas, après épreuve et pendant 6 mois, les titres neutralisants séri- ques sont compris entre $1 / 10^{\mathrm{e}}$ et $1 / 20^{\mathrm{e}}$. Vers le $8^{\mathrm{e}}$ et $9^{\mathrm{e}}$ mois, il y a une chute : moyenne située entre $1 / 5^{\circ}$ et $1 / 10^{\mathrm{e}}$.

2. Essai d'interprétation : relation entre immunité et pouvoir neutralisant des sérums

On constate un certain parallèlisme entre l'évolution des titres neutralisants des sérums, et d'autre part, le degré de résistance des agneaux vaccinés à l'infection expérimentale.

Les résultats de cette expérimentation sont trop limités pour que l'on puisse en tirer des conclusions générales. Toutefois un certain nombre de faits sont en faveur d'une immunité sérique reposant sur l'apparition des anticorps neutralisants :

L'absence de ces antlworps chez les agneaux neufs, la présence de ces anticorps chez les vaccinés et les éprouvés, l'évolution parallèle des anticorps et de l'indice de résistance à l'épreuve.

On peut noter également que, lorsqu'il n'y a pas de réaction locale inflammatoire post-vaccinale, les anticorps neutralisants sériques n'apparaissent pas et la résistance à l'épreuve est inexistante.

Qu'il s'agisse de vaccination ou d'épreuve, le niveau des anticorps neutralisants demeure relativement faible.

L'hypothèse d'une immunité de type sérique basée sur la présence d'anticorps neutralisants n'exclut évidemment pas l'intervention d'autres mécanismes de défense spécifique.

\section{CONCLUSION}

L'adaptation du virus claveleux aux cultures cellulaires est assez facile. Par contre l'atténuation d'une souche sauvage par passage en série en cultures cellulaires est un travail de longue durée dont le résultat n'est pas automatiquement assuré.

Le titre du virus claveleux de culture cellulaire n'est pas très élevé lorsqu'il s'exprime par l'effet cytopathogène. Par contre le titre infectieux sur mouton est nettement plus important. Il y a un rapport assez constant entre les deux. 
Le virus atténué de culture cellulaire est peu pathogène pour le mouton, encore qu'il y ait des variations de sensibilité selon les races. Le virus protège le mouton contre l'épreuve virulente et il fait apparaître des anticorps neutralisants dans le sérum. L'immunité dure au moins six mois.
L'immunité repose au moins en partie sur l'existence d'anticorps neutralisants sériques, dont le taux demeure assez faible. Pour qu'apparaissent les anticorps et l'état d'immunité, il faut que la vaccination soit suivie d'une réaction cutanée locale.

\section{SUMMARY \\ Culture of the sheep-pox virus on cells. Application to vaccination and immunity control}

In this paper, the properties of the sheep-pox virus cultured on cells are discussed : the cytopathogenic effect, titre, innocuousness, immunizing capacity, induction of specific serum antibodies.

\section{RESUMEN}

Cultivo del virus de la viruela ovina sobre células : aplicación a la vacunación y a la comprobación de la inmunidad

Se estudian algunas propiedades del virus de la viruela ovina cultivado sobre células in vitro: E. C. P., título, innocuidad, poder inmunizante, inducción de anticuerpos sericos específicos.

\section{BIBLIOGRAPHIE}

1. CHIFNEY (S. T.) et collab. Factors related to the production of attenuated vaccines against sheep-pox. Res. vet. Sci., 1973, 14 (1) : 62.

2. CILLI (V.) et BALDELLI (B.). Il virus del vaiolo ovino su cellule testicolari de ovis aries cultivate in vitro. Bull. Ist. Seroterap. Milanese, 1957, 36:611.

3. LANG (M.) et LEFTHERIOTIS (E.). Adaptation du virus de la clavelée sur les cellules rénales de mouton. Bull. Acad. vét. Fr., 1961, $34: 367$.

4. MATEVA-PENKOVA (V.) et collab. Sheep-pox vırus and its use as a vaccine. Bull. O. I. E., 1974, 81 (3-4) : 329.
5. PLOWRIGHT (W.) et FERRIS (R.). The growth and cytopathogenicity of sheep-pox virus in tissue cultures. Brit. J. exp. Path., 1968, $39: 424$.

6. RAMYAR (H.) et collab. Observation on the use of live modified tissue culture vaccine against sheep-pox. Bull. O.I.E., 1974, 81 (9-10) : 881. Arch. Inst. Razi., 1976, 28 : 11-16.

7. RAMYAR (H.) et collab. La variole caprine : valeur immunigène du virus vaccin modifié sur cultures cellulaires. Recl. méd. vét., 1974, 150 (2) : 131. 